\title{
Outcomes of patients with heart failure after primary prevention ICD unit generator replacement
}

\author{
Khang-Li Looi, ${ }^{\oplus}$ Andrew Gavin, ${ }^{2}$ Lisa Cooper, ${ }^{1}$ Liane Dawson, ${ }^{2}$ Debbie Slipper, ${ }^{2}$ \\ Nigel Lever ${ }^{1,3}$
}

${ }^{1}$ Green Lane Cardiovascular Service, Auckland City Hospital, Auckland, New Zealand ${ }^{2}$ Cardiovascular Division, North Shore Hospital, Auckland, New Zealand

${ }^{3}$ Department of Medicine, University of Auckland, Auckland, New Zealand

\section{Correspondence to}

Dr Khang-Li Looi, Green Lane Cardiovascular Service, Auckland City Hospital, Auckland 1023, New Zealand; khanglil@adhb. govt.nz

Received 4 December 2018 Revised 13 December 2018 Accepted 30 December 2018

\section{ABSTRACT}

Objective Data describing outcomes after implantable cardioverter-defibrillator (ICD) unit generator replacement in patients with heart failure (HF) with primary prevention devices are limited.

Method Data on patients with HF who underwent primary prevention ICD/cardiac resynchronisation therapy-defibrillator (CRT-D) implantation from 2007 until mid-2015 who subsequently received unit generator replacement were analysed. Outcomes assessed were mortality, appropriate ICD therapy and shock, and procedural complications.

Results 61 of 385 patients with HF with primary prevention ICD/CRT-D undergoing unit generator replacement were identified. Follow-up period was $1.8 \pm 1.5$ years after replacement. $43(70.5 \%)$ patients had not received prior appropriate ICD therapy prior to unit replacement. The cumulative risks of appropriate ICD therapy at 1,3 and 5 years after unit replacement in those without prior ICD therapy were $0 \%, 6.2 \%$ and $50 \%$ compared with $6.2 \%, 59.8 \%$ and $86.6 \%$, respectively $(p=0.005)$ in those with prior ICD therapies. No predictive factors associated with appropriate ICD therapy after replacement could be identified. 41 (32.8\%) patients no longer met guideline indications at the time of unit replacement but risks of subsequent appropriate ICD interventions were not different compared with those who continued to meet primary prevention ICD indications.

The 5-year mortality risk after unit replacement was $18.4 \%$ and there were high procedural complication rates $(9.8 \%)$.

Conclusion No predictive marker successfully stratified patients no longer needing ICD support prospectively. Finding such a marker is important in decision-making about device replacement particularly given the concerns about the complication rates. These factors should be considered at the time of ICD unit replacement.

\section{INTRODUCTION}

Implantable cardioverter-defibrillators (ICD) and cardiac resynchronisation therapy-defibrillators (CRT-D) are standard treatments for the prevention of sudden cardiac death (SCD) in selected patients with heart failure (HF). ${ }^{1}$ Despite advances in technology, the majority of ICD or CRT-D patients outlive their device and have to undergo one or more unit generator replacements. ${ }^{2}$ Registry data have shown that only one-fourth of primary prevention patients will experience appropriate ICD therapy during the initial unit generator service life (or lifespan). ${ }^{34}$ This means that approximately

\section{Key messages}

What is already known about this subject?

- The current published guidelines do not distinguish between patients receiving initial implantable cardioverter-defibrillators (ICD) and those undergoing elective unit generator replacement.

- A debate continues on how to approach patients implanted with primary prevention ICDs referred for elective replacement due to battery depletion.

What does this study add?

- Patients developed significant comorbidities during follow-up and there were high procedural complication rates in those undergoing elective unit generator replacement.

- There were no predictors for those at lower risk of needing ICD therapy identified.

How might this impact on clinical practice?

- Discussion with ICD patients about life expectancy and accumulated comorbidity is important and required during time of elective replacement.

- This will allow each patient to choose in advance what interventions they wish to receive as part of end-of-life care.

$75 \%$ of patients have not required ICD therapy by the time of unit replacement.

The risk/benefit ratio of ICDs varies over time and should be re-evaluated at the time of replacement. Whether these patients require device replacement is still a matter of debate. There are many gaps in the current knowledge related to optimal ICD replacement strategy. A comprehensive assessment of the overall benefits and implications of ongoing ICD therapy should be undertaken based on a patient's needs and preferences. Shared decision-making needs to take place allowing valid informed consent prior to the implantation process.

The New Zealand Cardiac Implanted Device Registry has recently been developed on the All New Zealand Acute Coronary Syndrome-Quality Improvement platform. ${ }^{5}$ The first description of data on new pacemaker implants from this Registry has been recently published. ${ }^{5}$ Data on ICD implants are still pending. The aim of our study was to determine the clinical characteristics and outcomes of patients with HF in the Northern Region of New 
Zealand implanted with primary prevention ICD/CRT-Ds who underwent unit generator replacement.

\section{METHOD}

This was a retrospective observational study.

The Northern Region of New Zealand is defined as the four northernmost District Health Board (DHB) areas and consists of Auckland DHB, Counties Manukau DHB, Northland DHB and Waitemata DHB. The four DHBs in the Northern Region serve $38 \%$ of the total New Zealand population with an estimated 1.76 million people in this region. ${ }^{6}$ We have previously reported the long-term outcomes of 385 patients with HF in the Northern Region of New Zealand with primary prevention ICD/CRT-D implanted between 2007 and mid-2015. ${ }^{7}$ This group of patients was followed up to end of year 2017 until they reached elective replacement of the unit generator due to battery depletion.

Data collection included patient characteristics, type of device implanted, left ventricular ejection fraction (LVEF), renal function using estimated glomerular filtration rate (eGFR) and the presence of comorbidities at baseline and at the time of unit generator replacement. Echocardiographic assessments were used to assess LVEF prior to ICD generator change. Comorbidities of interest included neoplastic disease, atrial fibrillation (AF) and history of transient ischaemic attack/stroke. Pertinent medication use (beta-blockers, ACE inhibitors or angiotensin receptor blockers (ARB) and antiarrhythmic drugs) at baseline and at the time of unit generator replacement was reviewed. Data were also collected from device interrogation records, which included delivery of appropriate therapies (shock or antitachycardia pacing (ATP) for ventricular arrhythmia) and inappropriate therapies (shock or ATP for non-ventricular arrhythmia event). Programming the new ICD unit generator was based on the published expert consensus statement on optimal ICD programming and testing. ${ }^{8}$

\section{Statistical analysis}

Baseline characteristics were summarised as either mean \pm SD or frequency with percentage depending on the nature of the data. The paired sample t-test was used for comparison of continuous variables and the $\chi^{2}$ test was used for comparing frequencies between the groups where appropriate. Survival rates over time were depicted in Kaplan-Meier curves, and the differences between survival distributions were evaluated with the log-rank test. Multivariable logistic regression analysis was performed to identify potential clinical predictors of appropriate ICD therapy and death after unit generator replacement.

Statistical analyses were performed using SPSS V.25.0 (IBM Software). All statistical tests were two sided. P values $<0.05$ were considered statistically significant.

\section{RESULTS}

From year 2007 to mid-2015, a total of 385 patients with HF were implanted with primary prevention ICD or CRT-D. The mean follow-up was $7.7 \pm 2.2$ years after the initial implantation. At the end of follow-up, 95 (24.7\%) patients had died and 20 (5.2\%) patients had been lost to follow-up as they had moved out of the Northern Region. During the follow-up, 15 (3.9\%) patients had had their ICD/CRT-D devices deactivated: 10 deactivated the devices prior to their deaths and the remaining five patients had had their device deactivated as part of their advanced care planning (ACP). Fifteen (3.9\%) patients had their devices removed during orthotopic cardiac transplant.
Table 1 Characteristics of patients at initial ICD/CRT-D implantation and at the time of ICD/CRT-D replacement

\begin{tabular}{|c|c|c|c|}
\hline & $\begin{array}{l}\text { Initial } \\
\text { implantation } \\
(n=61)\end{array}$ & $\begin{array}{l}\text { Generator } \\
\text { replacement } \\
(n=61)\end{array}$ & $P$ value \\
\hline Mean age & $58.2 \pm 11.4$ & $64.1 \pm 12.1$ & $<0.01$ \\
\hline Median age & $60.3(15.8)$ & $65.9(18.7)$ & \\
\hline Mean LVEF (\%) & $24.8 \pm 5.2$ & $31.2 \pm 11.6$ & $<0.01$ \\
\hline \multicolumn{4}{|l|}{ Type of device } \\
\hline \multicolumn{4}{|l|}{$I C D$} \\
\hline Single chamber & 22 & 22 & NA \\
\hline Dual chamber & 9 & 9 & \\
\hline Subcutaneous ICD (S-ICD) & 4 & 4 & \\
\hline CRT-D & 26 & 26 & \\
\hline \multicolumn{4}{|l|}{ NYHA heart failure class (\%) } \\
\hline Class I & $16(26.2)$ & $23(37.7)$ & 0.08 \\
\hline Class II & $32(52.5)$ & $33(54.1)$ & \\
\hline Class III & $13(21.3)$ & $4(6.6)$ & \\
\hline Class IV & 0 & $1(16)$ & \\
\hline Mean eGFR $\left(\mathrm{mL} / \mathrm{min} / 1.73 \mathrm{~m}^{2}\right)$ & $70.3 \pm 16.7$ & $65.3 \pm 19.2$ & 0.02 \\
\hline History of atrial fibrillation (\%) & $15(24.6)$ & $23(37.7)$ & 1.0 \\
\hline History of TIA/stroke (\%) & $4(6.6)$ & $6(9.8)$ & 0.53 \\
\hline Neoplastic disease (\%) & 0 & $1(1.6)$ & 0.32 \\
\hline Diabetes mellitus (\%) & $12(19.7)$ & $16(26.2)$ & 0.04 \\
\hline Hypertension (\%) & $14(22.9)$ & $16(26.2)$ & 0.42 \\
\hline \multicolumn{4}{|l|}{ Medications use } \\
\hline Diuretics (\%) & 39 (63.9) & $37(60.7)$ & 0.57 \\
\hline ACE inhibitor or ARB (\%) & $58(95.1)$ & $55(90.2)$ & 0.26 \\
\hline Beta-blocker (\%) & $53(86.9)$ & $55(90.2)$ & 0.48 \\
\hline \multicolumn{4}{|l|}{ Antiarrhythmic drugs } \\
\hline Sotalol (\%) & $7(11.5)$ & $2(3.3)$ & 0.16 \\
\hline Amiodarone (\%) & $8(13.1)$ & $13(21.3)$ & 0.17 \\
\hline
\end{tabular}

TIA, transient ischaemic attackARB, angiotensin receptor blocker; CRT-D, cardiac resynchronisation therapy-defibrillator; ICD, implantable cardioverter-defibrillator; LVEF, left ventricular ejection fraction; NYHA, New York Heart Association; eGFR, estimated glomerular filtration rate.

Two patients with no pacing indications had devices extracted due to sepsis and declined further reimplantation. One patient died prior to the scheduled unit generator replacement and two patients with no pacing indications decided against replacement after comprehensive medical evaluation and discussion.

Only 61 patients underwent unit generator replacement during the follow-up period. The majority were male $(n=50$, $81.9 \%)$ and of European descent $(n=39,63.9 \%)$. These patients were more likely to have non-ischaemic cardiomyopathy (NICM) $(n=38,62.3 \%)$. ICD was the most common device replaced $(n=39,63.9 \%)$, and the majority of the ICDs were single-chamber devices (56.4\%). CRT-D comprised $36.1 \%$ device replaced. The mean time between the initial implantation and unit generator replacement was $5.83 \pm 2.0$ years. The mean longevity was $5.8 \pm 1.9$ years for single-chamber devices, $6.1 \pm 1.4$ years for dual-chamber devices, $5.7 \pm 0.4$ years for subcutaneous ICD and $5.5 \pm 1.6$ years for CRT-D devices $(p=0.24)$.

Table 1 showed the baseline characteristics of patients at the time of the initial implantation and at the time of generator replacement. At the time of generator replacement, patients had a significantly higher LVEF $(31.2 \pm 11$. vs $24.8 \pm 5.2, \mathrm{p}<0.01)$, higher prevalence of diabetes mellitus $(p=0.04)$ and lower mean eGFR ( $p=0.02$ ) but no other significant differences were found. One patient downgraded from CRT-D to CRT-pacemaker at the time of replacement as there was significant improvement 
Total Cohort $(n=61)$

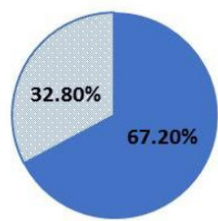

ICD cohort $(n=39)$

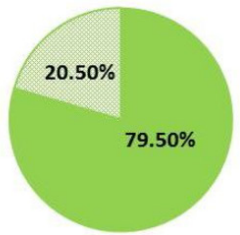

CRT-D cohort $(n=22)$

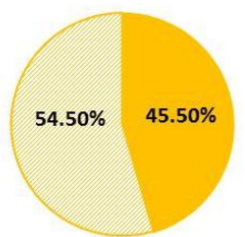

- ICD indicated $\square$ ICD not indicated

ICD indicated $\square$ ICD not indicated

ICD indicated $\square$ ICD not indicated

Figure 1 Implantable cardioverter-defibrillator (ICD) indications at elective unit generator replacement. CRT-D, cardiac resynchronisation therapydefibrillator.

in LVEF. One patient with no previous ICD therapy downgraded from dual-chamber ICD to pacemaker at the time of unit generator replacement as part of ACP. For 21 (34.4\%) patients receiving unit generator replacement, an upgrade/lead addition was also performed.

\section{Indications and predictors of continued ICD use at unit generator replacement}

Forty-one (67.2\%) patients fulfilled the guideline criteria based on LVEF for continued ICD use at unit generator replacement (figure 1).

Among the eight ICD patients who did not meet the guideline criteria, 50\% had received appropriate ICD therapy in the intervening years despite improvement in LVEF at the time of unit generator replacement. For those with CRT-D, 45.4\% fulfilled the guideline criteria for replacement. The other 54.5\% had demonstrated improvement in LVEF to $\geq 40 \%$ and only one patient had received appropriate ICD therapy prior to unit generator replacement.

Characteristics of patients who continued to meet criteria for an ICD at the time of replacement versus those who no longer met criteria are compared in table 2 . No differences in terms of mean age and ethnicity were noted in the two groups. However, there were significantly higher LVEF, more females, more patients with CRT-D use and with NICM as well as higher use of ACE inhibitors/ARBs and beta-blockers in those who did not meet guideline criteria.

\section{Incidence and predictors of appropriate ICD therapy after unit generator replacement}

Among the 61 patients who underwent generator replacement, $18(29.5 \%)$ had received prior appropriate ICD therapy. Baseline characteristics of those with or without prior appropriate ICD therapy are shown in table 3 . Those with prior ICD therapy were more likely to be male and more often treated with diuretics and amiodarone at the time of unit generator replacement.

The mean follow-up was $1.8 \pm 1.5$ years after unit generator replacement. During follow-up, $13(21.3 \%)$ of the 61 patients received appropriate ICD therapy (ATP and/or ICD shocks) after unit replacement. The mean time from unit replacement to appropriate ICD therapies was $0.9 \pm 0.9$ years. As expected, the occurrence of appropriate ICD therapy after ICD generator replacement was considerably higher for patients who met the guideline criteria for ICD (table 2) and those with prior ICD therapies (table 3). The cumulative risks of appropriate ICD interventions at 1,3 and 5 years after generator replacement in those with prior ICD therapy were $6.2 \%, 59.8 \%$ and $86.6 \%$ and in those without prior ICD therapies were $0 \%, 6.2 \%$ and
$50 \%$, respectively, log-rank $\mathrm{p}=0.005$ (figure 2 ). No predictive factors for lower need of ICD therapy could be identified in either groups.

For the 20 patients who underwent ICD device replacement (despite no longer meeting accepted indications for primary prevention ICD therapy), 10\% received appropriate ICD therapies compared with $26.8 \%$ who continued to meet primary prevention ICD indications $(\mathrm{p}<0.01)$. The cumulative risks of appropriate ICD interventions at 1,3 and 5 years after unit generator replacement in those who no longer met indications

Table 2 Characteristics of patients who met or did not meet the criteria for primary prevention ICD at the time of unit generator replacement

\begin{tabular}{|c|c|c|c|}
\hline & $\begin{array}{l}\text { Met guideline } \\
\text { criteria }(n=41)\end{array}$ & $\begin{array}{l}\text { Did not meet } \\
\text { guideline } \\
\text { criteria }(n=20)\end{array}$ & $P$ value \\
\hline \multicolumn{4}{|l|}{ Gender (\%) } \\
\hline Male & $36(87.8)$ & $14(70)$ & \multirow[t]{2}{*}{0.02} \\
\hline Female & $5(12.2)$ & $6(30)$ & \\
\hline \multicolumn{4}{|l|}{ Type of device (\%) } \\
\hline $\mathrm{ICD}$ & $31(75.6)$ & $8(40)$ & \multirow[t]{2}{*}{0.04} \\
\hline CRT-D & $10(24.4)$ & $12(60)$ & \\
\hline \multicolumn{4}{|l|}{ Cardiomyopathy (\%) } \\
\hline ICM & $17(41.5)$ & $2(10)$ & $<0.01$ \\
\hline $\mathrm{NICM}$ & $21(51.2)$ & $17(85)$ & \\
\hline Other causes & $3(7.3)$ & $1(5)$ & \\
\hline Mean LVEF (\%) & $24.7 \pm 5.4$ & $44.5 \pm 9.2$ & 0.01 \\
\hline Mean eGFR (mL/min/1.73 $\left.\mathrm{m}^{2}\right)$ & $62.6 \pm 17.6$ & $67.6 \pm 21.5$ & 0.19 \\
\hline Hypertension (\%) & $12(29.3)$ & $4(20)$ & 0.11 \\
\hline Diabetes mellitus (\%) & $12(29.3)$ & $4(20)$ & 0.11 \\
\hline Atrial fibrillation (\%) & $16(39)$ & $8(40)$ & 0.89 \\
\hline History of stroke/TIA (\%) & $5(12.2)$ & $1(5)$ & 0.07 \\
\hline \multicolumn{4}{|c|}{ Medications use at generator replacement } \\
\hline ACE inhibitor or ARB (\%) & $35(85.3)$ & $20(100)$ & $<0.01$ \\
\hline Beta-blocker (\%) & $35(85.3)$ & $20(100)$ & $<0.01$ \\
\hline \multicolumn{4}{|l|}{ Antiarrhythmic drugs } \\
\hline Amiodarone (\%) & $9(21.9)$ & $4(20)$ & 0.44 \\
\hline $\begin{array}{l}\text { ICD therapy prior to generator } \\
\text { replacement }(\%)\end{array}$ & $13(31.7)$ & $5(25)$ & 0.27 \\
\hline $\begin{array}{l}\text { ICD therapy after generator } \\
\text { replacement }(\%)\end{array}$ & $11(26.8)$ & $2(10)$ & $<0.05$ \\
\hline \multicolumn{4}{|c|}{$\begin{array}{l}\text { ARB, angiotensin receptor blocker; CRT-D, cardiac resynchronisation therapy-defibrillator } \\
\text { ICD, implantable cardioverter-defibrillator; ICM, ischaemic cardiomyopathy; LVEF, left } \\
\text { ventricular ejection fraction; NICM, non-ischaemic cardiomyopathy; TIA, transient } \\
\text { ischaemic attack; eGFR, estimated glomerular filtration rate. }\end{array}$} \\
\hline
\end{tabular}


Table 3 Baseline clinical and demographic characteristics of patients with and without prior ICD therapy

\begin{tabular}{|c|c|c|c|}
\hline Characteristic & $\begin{array}{l}\text { Patients with } \\
\text { prior ICD } \\
\text { therapy }(n=18)\end{array}$ & $\begin{array}{l}\text { Patients } \\
\text { without prior } \\
\text { ICD therapy } \\
(n=43)\end{array}$ & $\mathrm{p}$ value \\
\hline Mean age at implantation & $59.9 \pm 9.3$ & $57.5 \pm 12.1$ & 0.42 \\
\hline Mean age at replacement & $66.1 \pm 9.5$ & $63.2 \pm 13$ & 0.29 \\
\hline \multicolumn{4}{|l|}{ Gender (\%) } \\
\hline Male & $17(94.4)$ & $33(76.7)$ & \multirow[t]{2}{*}{$<0.01$} \\
\hline Female & $1(5.6)$ & $10(23.3)$ & \\
\hline \multicolumn{4}{|l|}{ Type of device (\%) } \\
\hline ICD & $13(72.2)$ & $26(60.5)$ & \multirow[t]{2}{*}{0.05} \\
\hline CRT-D & $5(27.8)$ & $17(39.5)$ & \\
\hline \multicolumn{4}{|l|}{ Cardiomyopathy (\%) } \\
\hline ICM & $7(38.9)$ & $12(27.9)$ & \multirow[t]{3}{*}{0.81} \\
\hline NICM & $11(61.1)$ & $27(62.8)$ & \\
\hline Other causes & 0 & $4(9.3)$ & \\
\hline Mean LVEF at implant (\%) & $25.6 \pm 5.8$ & $24.6 \pm 5.0$ & 0.39 \\
\hline Mean LVEF at replacement (\%) & $27.6 \pm 8.3$ & $32 \pm 11.9$ & 0.13 \\
\hline Mean eGFR at implant & $61.8 \pm 9.1$ & $62.1 \pm 14.2$ & 0.12 \\
\hline Mean eGFR at replacement & $58.8 \pm 18.2$ & $66.4 \pm 19.3$ & 0.19 \\
\hline \multicolumn{4}{|l|}{ Medications at initial implant } \\
\hline Diuretics (\%) & $15(83.3)$ & $22(51.2)$ & $<0.01$ \\
\hline ACE inhibitor/ARB (\%) & $18(100)$ & $38(88.4)$ & 0.02 \\
\hline Beta-blocker (\%) & $14(77.8)$ & $37(86)$ & 0.01 \\
\hline Amiodarone (\%) & $3(16.7)$ & $5(11.6)$ & 0.29 \\
\hline \multicolumn{4}{|l|}{ Medications at replacement } \\
\hline Diuretics (\%) & $13(72.2)$ & $24(55.8)$ & $<0.01$ \\
\hline ACE inhibitor/ARB (\%) & $17(94.4)$ & $38(88.4)$ & 0.14 \\
\hline Beta-blockers (\%) & $15(83.3)$ & $40(93)$ & 0.03 \\
\hline Sotalol (\%) & $1(5.6)$ & $1(2.3)$ & 0.32 \\
\hline Amiodarone (\%) & $9(50)$ & $4(9.3)$ & $<0.01$ \\
\hline $\begin{array}{l}\text { ICD therapy after replacement } \\
(\%)\end{array}$ & $10(55.6)$ & $3(7)$ & $<0.01$ \\
\hline ATP & 10 & 3 & $<0.01$ \\
\hline ICD shocks & 7 & 1 & $<0.01$ \\
\hline $\begin{array}{l}\text { Inappropriate ICD therapy after } \\
\text { replacement (\%) }\end{array}$ & 0 & $1(2.3)$ & 0.19 \\
\hline $\begin{array}{l}\text { Number of death after replacement } \\
(\%)\end{array}$ & $1(5.6)$ & $4(9.3)$ & 0.33 \\
\hline
\end{tabular}

ARB, angiotensin receptor blocker; ATP, antitachycardia pacing; CRT-D, cardiac resynchronisation therapy-defibrillator; ICD, implantable cardioverter-defibrillator; ICM, ischaemic cardiomyopathy; LVEF, left ventricular ejection fraction; NICM, non-ischaemic cardiomyopathy; eGFR, estimated glomerular filtration rate.

were $0 \%, 25 \%$ and $62.5 \%$ and in those who continued to meet primary prevention ICD indications were $3 \%, 28.4 \%$ and $85.9 \%$, respectively, log-rank $\mathrm{p}=0.23$ (figure 3 ).

\section{Complications}

There were a total of six $(9.8 \%)$ procedure-related complications with three $(50 \%)$ of these complications occurring in patients undergoing additional transvenous lead during replacement or upgrade to CRT-D. Early complications $(<30$ days after unit generator replacement) occurred in one $(1.6 \%)$ patient who developed haematoma that was treated conservatively on an outpatient basis. Other complications (8.2\%) (>30 days) included the need for reoperation resulting from lead malfunction due to increasing threshold and poor sensing in four patients $(6.6 \%)$ and infection requiring intravenous antibiotics in one patient $(1.6 \%)$.

\section{Mortality}

A total of five patients (8.2\%) died during follow-up after unit generator replacement, all from end-stage HF. The mean time from death after unit generator replacement was $1.1 \pm 1.3$ years. The 1, 3 and 5-year mortality risk was 5.2\%, 8.2\% and 18.4\%, respectively. During follow-up, one $(5.6 \%)$ of the 18 patients who received prior ICD therapy died after unit generator replacement. Of 48 patients with no prior ICD therapy, four $(8.3 \%)$ died after unit generator replacement. There was no difference in mortality rate between the two groups (log-rank $\mathrm{p}=0.57$, HR 5.14; 95\% CI 4.54 to $5.75, \mathrm{p}=0.33$ ) (figure 4). No predictors of survival were identified.

\section{DISCUSSION}

Unsurprisingly, we found that patients who had previously undergone primary prevention ICD/CRT-D implantation were likely to develop significant comorbidities during follow-up until the time of elective ICD/CRT-D unit generator replacement. Although $32.8 \%$ of patients no longer met primary prevention guideline indications at the time of unit generator replacement, the risks of subsequent appropriate ICD interventions were not different compared with those who continued to meet primary prevention ICD indications. 21.3 per cent of patients received appropriate ICD therapy after unit generator replacement and the occurrence of appropriate ICD therapy was considerably higher for those with prior ICD interventions. However, there were no predictors found to stratify those at lower risk of needing ICD therapy, in whom avoidance of ongoing ICD support might have been an option.

Current guidelines do not distinguish between de novo ICD implantation and those undergoing elective unit generator replacement. The study by Kini et al showed that approximately $26 \%$ of patients who received primary prevention ICDs no longer met guideline-driven indications for an ICD at the time of unit generator replacement. Furthermore, these patients had a significantly lower rate of subsequent ICD therapies. ${ }^{9}$ Our study shows that a significant proportion $(32.8 \%)$ of patients who receive their initial ICD for primary prevention on the basis of a low LVEF undergo unit generator replacement despite improved LVEF and not requiring ICD therapy in the intervening years. However, the cumulative risks of appropriate ICD interventions after unit generator replacement were no different from those with ongoing indications. This may be explained by the small number of patients, and our results would be consistent with lower subsequent ICD therapy rate observed in those no longer meeting guideline-driven indications.

There is a general trend towards replacing ICD regardless of the patient's clinical evolution during the device lifetime. In the results of the European Heart Rhythm Association survey, the overwhelming majority of centres in Europe reported that they replaced ICDs at the end of battery life. ${ }^{10}$ Only in a small subset $(<10 \%)$ of patients with ICD for primary prevention and without ventricular arrhythmias since implantation, ICD was not replaced. ${ }^{10}$ Similar to our study, $32.8 \%$ of the patients who no longer met the guidelines for primary prevention ICD had their ICD replaced at the end of battery life. Only two patients required appropriate ICD therapies in this group after unit generator replacement. Patients without ICD therapies are at significantly lower risk of ICD therapies after unit generator replacement, especially if the LVEF has improved, and the risk of appropriate ICD therapy and/or rapid ventricular arrhythmia, although persisting over time, decreases significantly over the years. ${ }^{11}$ Shared discussions should occur with patients about the 
Freedom from ICD Therapies

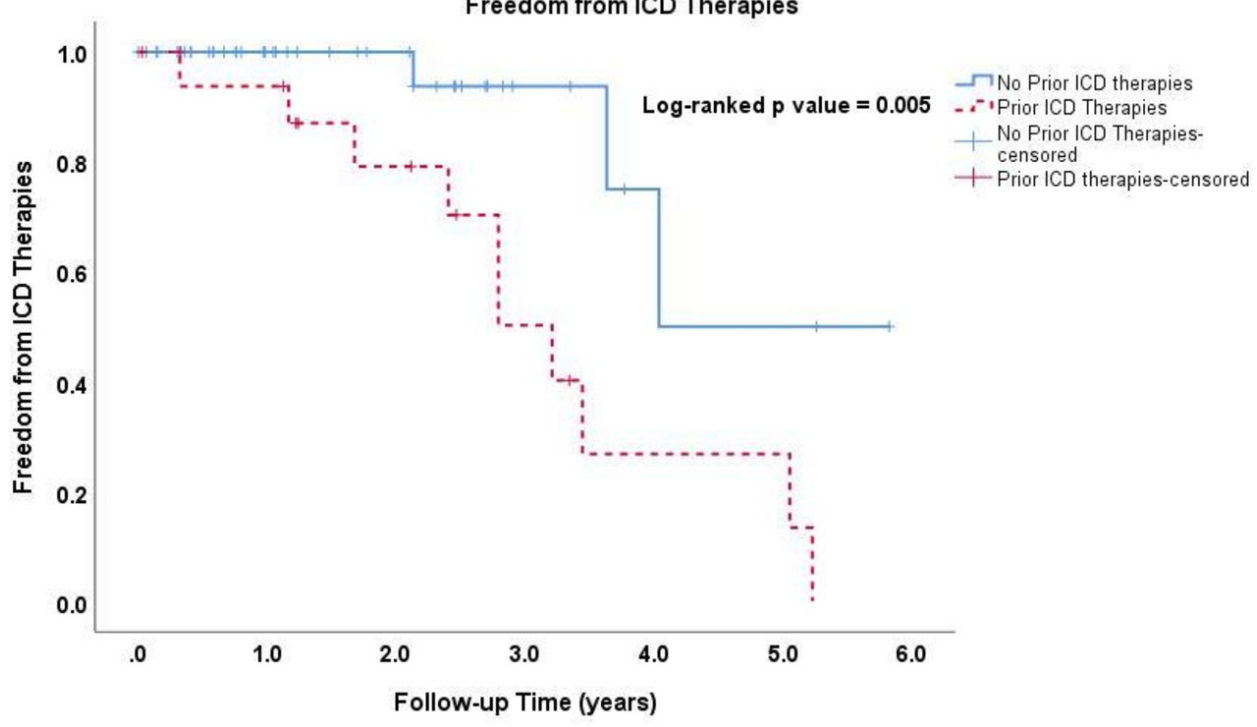

Number At Risk

\begin{tabular}{|l|l|l|l|l|l|l|l|}
\hline Year & $\mathbf{0}$ & $\mathbf{1}$ & $\mathbf{2}$ & $\mathbf{3}$ & $\mathbf{4}$ & $\mathbf{5}$ & $\mathbf{6}$ \\
\hline Prior ICD Therapies & 18 & 14 & 9 & 4 & 2 & 1 & 0 \\
\hline No Prior ICD Therapies & 43 & 24 & 16 & 6 & 2 & 1 & 0 \\
\hline
\end{tabular}

Figure 2 Subsequent implantable cardioverter-defibrillator (ICD) therapies after elective unit generator replacement in patients with prior ICD therapies compared with those without prior ICD therapies.

evidence, healthcare goals, risk tolerances and feelings about life and death trade-offs to enable high-quality decisions about ICD replacement. ICD unit generator replacement is an ideal time to re-evaluate healthcare goals and explore personal preferences regarding continuing ICD therapy. However, not many healthcare professions feel comfortable about this discussion. Furthermore, many patients due for replacement of their ICD unit generator did not realise they could opt out and many underestimated the risks of ICD replacement. A study by Lewis et al showed that $51.9 \%$ of the patients did not know that ICD unit generator replacement was optional. ${ }^{12}$ Of these, $27 \%$ would have considered no replacement. In the study, $20 \%$ of the patients believed ICD replacement carried no surgical risk and $17 \%$ perceived there was no risk of surgical infection. ${ }^{12}$ Therefore, it

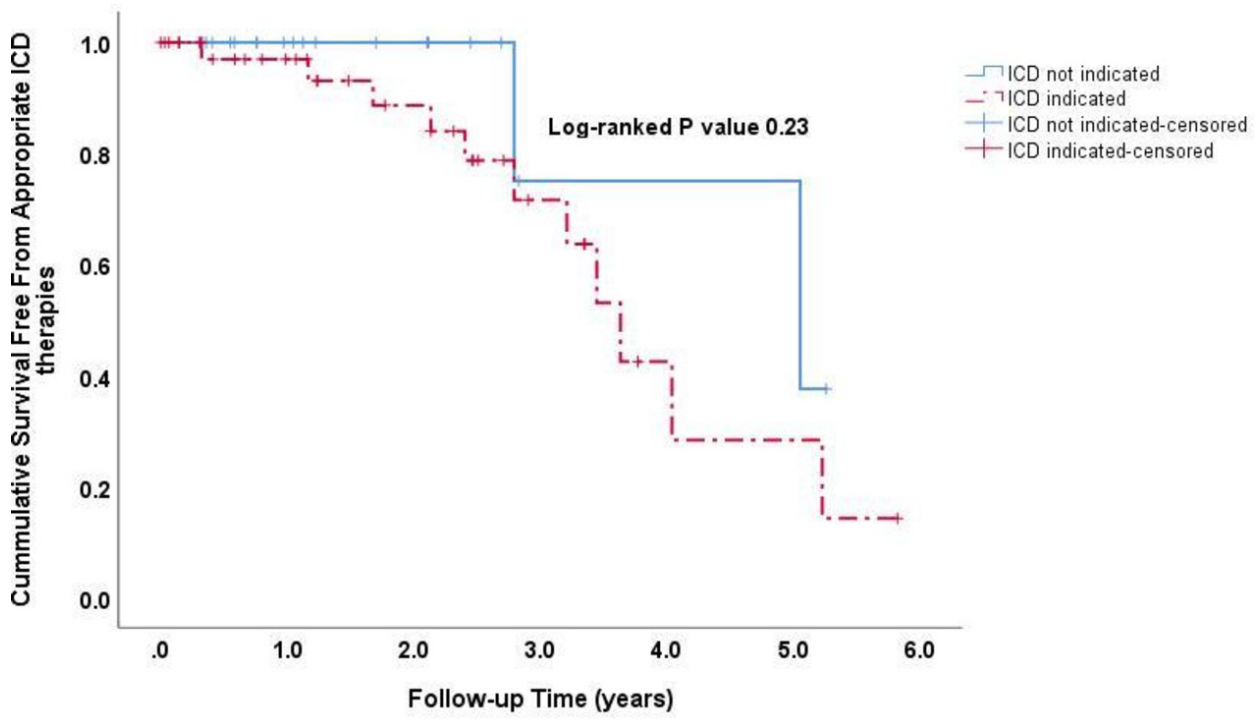

Number At Risk
\begin{tabular}{|l|l|l|l|l|l|l|l|}
\hline Year & $\mathbf{0}$ & $\mathbf{1}$ & $\mathbf{2}$ & $\mathbf{3}$ & $\mathbf{4}$ & $\mathbf{5}$ & $\mathbf{6}$ \\
\hline ICD not indicated & 20 & 11 & 7 & 2 & 1 & 1 & 0 \\
\hline ICD indicated & 41 & 27 & 18 & 8 & 2 & 1 & 0 \\
\hline
\end{tabular}

Figure 3 Subsequent implantable cardioverter-defibrillator (ICD) therapies after elective unit generator replacement in patients with no ICD indications compared with patients with ICD indications. 


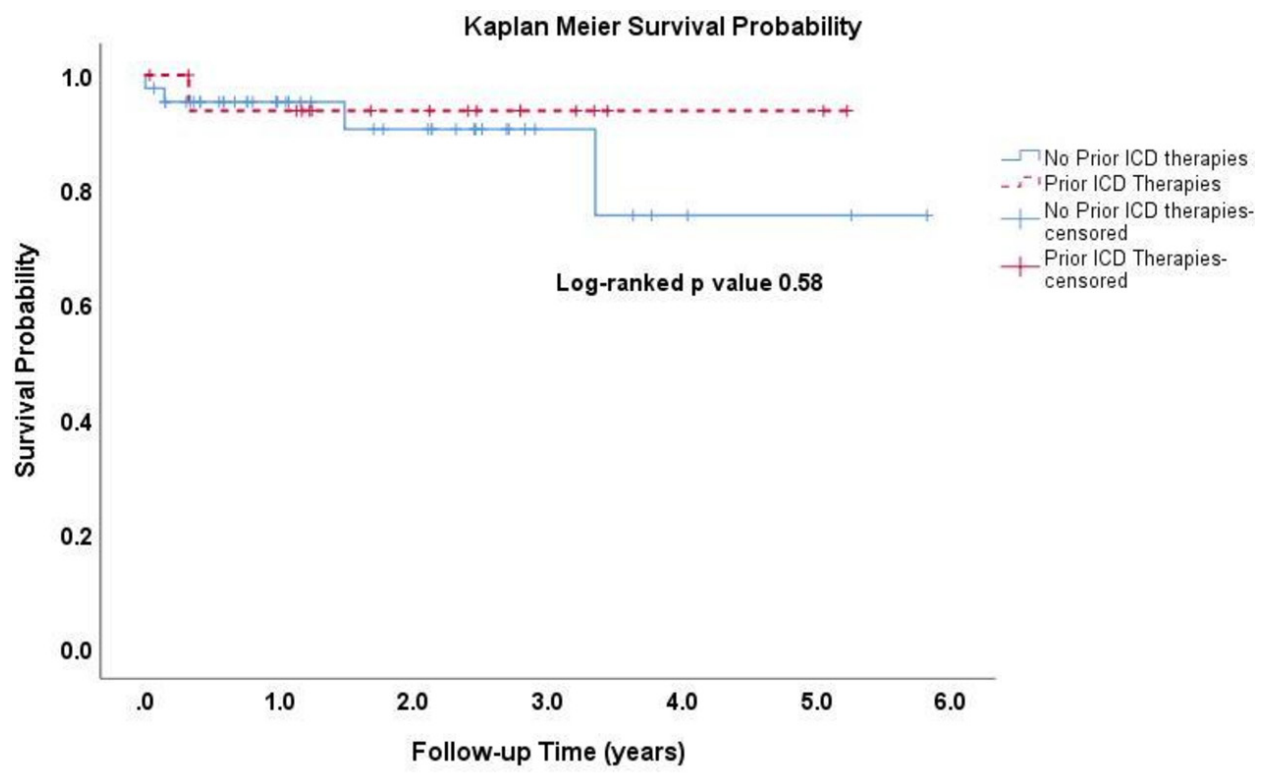

Number At Risk

\begin{tabular}{|l|l|l|l|l|l|l|l|}
\hline Year & $\mathbf{0}$ & $\mathbf{1}$ & $\mathbf{2}$ & $\mathbf{3}$ & $\mathbf{4}$ & $\mathbf{5}$ & $\mathbf{6}$ \\
\hline Prior ICD Therapies & 18 & 14 & 9 & 4 & 2 & 1 & 0 \\
\hline No Prior ICD Therapies & 43 & 23 & 16 & 6 & 2 & 1 & 0 \\
\hline
\end{tabular}

Figure 4 Kaplan-Meier survival curve in patients with prior implantable cardioverter-defibrillator (ICD) therapies compared with patients without prior ICD therapies.

is important to discuss risk and benefits before deciding whether to continue with ICD therapy as such a discussion would allow a patient and the treating physician to review their healthcare goals and reassess their views on risk and benefits. By exploring these topics, physicians can better integrate patient preference into decision-making.

Although primary prevention patients are at risk of developing ventricular arrhythmias, studies showed that only $35 \%$ receive appropriate ICD therapy for ventricular tachycardia or ventricular fibrillation. ${ }^{1314}$ Therefore, a significant number of patients have not developed ventricular arrhythmias requiring therapy during the first ICD service life. This raises the issue of whether or not such patients actually need a replacement. In two large ICD registries of all primary prevention patients who underwent first ICD unit generator replacement without prior ICD therapies, 11\% experienced appropriate ICD therapy during follow-up after unit generator replacement. ${ }^{15}$ Van Welsenes et al reported that although the majority of primary prevention ICD patients do not have ventricular arrhythmias during first battery service life, a substantial number of these patients subsequently get appropriate ICD therapy after replacement with a cumulative 5-year incidence for appropriate ICD therapy of 37\% (95\% CI $33 \%$ to $42 \%) .{ }^{16}$ The results of the Incidence Free Survival After ICD Replacement trial showed that at least $20 \%$ of patients without prior ICD therapies received appropriate ICD therapies within 3 years of unit generator replacement. ${ }^{3}$ Similarly, in our cohort, $21.3 \%$ of patients received appropriate ICD therapy after unit generator replacement and the 5 -year cumulative incidence of appropriate therapies was $29.9 \%$. No predictors of appropriate ICD therapy after replacement were identified in our study, precluding the ability to identify patients who may not benefit from ICD replacement. These results are consistent with previously published data. ${ }^{315}$
The results from the National Cardiovascular Data Registry ICD Registry showed that $>40 \%$ of patients died within 5 years following routine ICD unit generator replacement. ${ }^{17} \mathrm{AF}, \mathrm{HF}$ and LVEF were independently associated with poorer survival in patients following routine ICD unit generator replacement at the end of expected battery life. ${ }^{17}$ In addition, non-cardiac comorbidities including chronic lung disease, cerebrovascular disease, diabetes and worsening renal function were also independently associated with worse survival. ${ }^{17}$ At the time of generator replacement, our cohort patients had developed more comorbidities. Although no predictors of survival could be identified, the 1, 3 and 5-year mortality risk in our patients following ICD unit generator replacement was 5.2\%, $8.2 \%$ and $18.4 \%$, respectively. These findings underscore the importance of evaluating patients' entire clinical history at the time of ICD generator replacement, with particular attention to accumulated comorbidities that may limit life expectancy and the potential of benefiting from ongoing treatment with ICD therapy.

In our study, $24.7 \%$ of the patients had died prior to end of battery service life and 3.9\% patients had their ICD/CRT-D devices deactivated prior to their deaths or as part of ACP. HF is a chronic disease and can progressively deteriorate, resulting in poor long-term prognosis. ICDs improve prognosis by treating life-threatening ventricular arrhythmia, but do not modify HF progression nor contribute positively to quality of life of these patients. The 2013 British Heart Foundation guidelines state that the appropriateness of maintaining ICD therapy must be regularly reviewed as part of monitoring of the patient's progressive disease if there is any change in clinical status including the development of a life-limiting disease. ${ }^{18}$ Similarly, joint guidance from Resuscitation Council UK, Heart Rhythm UK/Arrhythmia Alliance and British Cardiovascular Society advises that patients 
approaching end of life should be offered a choice of ICD deactivation to avoid shocks in the latter stages of their illness. ${ }^{19}$

Careful decision-making before ICD replacement is important because there is a potential risk of complications related to ICD replacement. ${ }^{20}{ }^{21}$ In the Implantable Cardiac Pulse Generator Replacement (REPLACE) registry, there was a major complication rate of $4.0 \%$ in patients who had a generator replacement without a plan to add a transvenous lead. ${ }^{20}$ Complications were highest in patients who had an upgrade to or a revised CRT device (18.7\%; 95\% CI 15.1 to 22.6). ${ }^{20}$ Our cohort had a higher complication rate due to additional transvenous lead or upgrade, similar to published data that adverse event rates increase from pacemaker to ICD to CRT. ${ }^{2223}$

\section{Limitations}

Our study should be interpreted in light of its potential limitations. Our study cohort was retrospectively recruited but prospectively followed up. The numbers of patients in our study were small, and the number of patients followed out after 2 years was small. Our population of patients receiving primary prevention ICD was also generally younger than in most Western countries, especially the USA. Our study was observational and involved patients in the Northern Region of New Zealand, with a different socioeconomic and ethnicity mix and will be different from other regions in New Zealand. Given the paucity of data on ICD unit generator replacement in New Zealand, this study represents real-world data on the outcomes of these patients.

\section{CONCLUSION}

Most patients who undergo primary prevention ICD unit generator replacement in the Northern Region of New Zealand did not receive appropriate ICD therapy during the first generator longevity. One-third of patients who receive ICDs for primary prevention may no longer meet guideline criteria for continued ICD use at the time of elective unit generator replacement. However, these patients appear to be still at risk of SCD. Notably, there were no predictors for lower occurrence of ventricular arrhythmias. Our findings alone should not guide clinical decision-making for patients eligible for ICD replacement. However, these data may support ACP discussion with potential ICD patients about life expectancy and accumulated comorbidity as this will allow each patient to choose in advance what interventions they wish to receive as part of end-of-life care.

Acknowledgements The authors thank Charlene Nell, Desktop Support Team Administrator at Green Lane Cardiovascular Services/Cardiology Department for her secretarial support.

Contributors KLL was involved in conception and design of the study. KLL, LC, LD and DS were involved in data collection. KLL was involved in analysis and interpretation of data. KLL, AG and NL contributed to the writing of the manuscript as well as jointly developing the structure and arguments for the paper. All authors reviewed and approved the final manuscript.

Funding The authors have not declared a specific grant for this research from any funding agency in the public, commercial or not-for-profit sectors.

Competing interests None declared.

Patient consent for publication Not required.

Ethics approval The study protocol was approved by the Central Health and Disability Ethics Committee (Ethics ref: 15/CEN/58/AM02).

Provenance and peer review Not commissioned; externally peer reviewed.

\section{REFERENCES}

1. Priori SG, Blomström-Lundqvist C, Mazzanti A. 2015 ESC guidelines for the management of patients with ventricular arrhythmias and the prevention of sudden cardiac death: the task Force for the management of patients with ventricular arrhythmias and the prevention of sudden cardiac death of the European Society of cardiology (ESC). endorsed by: association for European paediatric and congenital cardiology (AEPC). Eur Heart J 2015;41:2793-867.

2. Biffi M, Ziacchi M, Bertini M, et al. How to truly value implantable cardioverterdefibrillators technology: up-front cost or daily cost? Int J Technol Assess Health Care 2011;27:201-6

3. Erkapic D, Sperzel J, Stiller S, et al. Long-term benefit of implantable cardioverter/ defibrillator therapy after elective device replacement: results of the INcidence free SUrvival after ICD REplacement (INSURE) trial--a prospective multicentre study. Eur Heart J 2013:34:130-7.

4. van Welsenes GH, van Rees JB, Borleffs CJW, et al. Long-term follow-up of primary and secondary prevention implantable cardioverter defibrillator patients. Europace 2011;13:389-94

5. Larsen PD, Kerr AJ, Hood M, et al. Pacemaker Use in New Zealand - Data From the New Zealand Implanted Cardiac Device Registry (ANZACS-QI 15). Heart Lung Circ 2016.

6. Statistics New Zealand. District Health boards ethnic group population projections, 2014-43 (2013-Base) - 2016 update. Wellington: Statistics New Zealand, 2016.

7. Looi K-L, Sidhu K, Cooper L, et al. Long-term outcomes of heart failure patients who received primary prevention implantable cardioverter-defibrillator: an observational study. J Arrhythm 2018;34:46-54.

8. Wilkoff BL, Fauchier L, Stiles MK, et al. 2015 HRS/EHRA/APHRS/SOLAECE expert consensus statement on optimal implantable cardioverter-defibrillator programming and testing. Europace 2016;18:159-83.

9. Kini V, Soufi MK, Deo R, et al. Appropriateness of primary prevention implantable cardioverter-defibrillators at the time of generator replacement: are indications still met? J Am Coll Cardiol 2014;63:2388-94.

10. Tilz R, Boveda S, Deharo JC, et al. Replacement of implantable cardioverter defibrillators and cardiac resynchronization therapy devices: results of the European heart rhythm association survey. Europace 2016;18:945-9.

11. Alsheikh-Ali AA, Homer M, Maddukuri PV, et al. Time-dependence of appropriate implantable defibrillator therapy in patients with ischemic cardiomyopathy. $J$ Cardiovasc Electrophysiol 2008;19:784-9.

12. Lewis $K B$, Nery PB, Birnie DH. Decision making at the time of ICD generator change: patients' perspectives. JAMA Intern Med 2014;174:1508-11.

13. Moss AJ, Greenberg H, Case RB, et al. Long-term clinical course of patients after termination of ventricular tachyarrhythmia by an implanted defibrillator. Circulation 2004;110:3760-5.

14. Borleffs CJ, van Rees JB, van Welsenes GH, et al. Prognostic importance of atrial fibrillation in implantable cardioverter-defibrillator patients. J Am Coll Cardiol 2010;55:879-85.

15. Yap S-C, Schaer BA, Bhagwandien RE, et al. Evaluation of the need of elective implantable cardioverter-defibrillator generator replacement in primary prevention patients without prior appropriate ICD therapy. Heart 2014;100:1188-92.

16. Van Welsenes GH, Van Rees JB, Thijssen J, et al. Primary prevention implantable cardioverter defibrillator recipients: the need for defibrillator back-up after an eventfree first battery service-life. J Cardiovasc Electrophysiol 2011:22:1346-50.

17. Kramer DB, Kennedy KF, Spertus JA, et al. Mortality risk following replacement implantable cardioverter-defibrillator implantation at end of battery life: results from the NCDR. Heart Rhythm 2014;11:216-21.

18. Beattie J. ICD deactivation at the end of life: principles and practice. London: British Heart Foundation, 2013

19. British Medical Association, Resuscitation Council (UK), The Royal College of Nursing. Decisions relating to cardiopulmonary resuscitation. 3rd ed. London: British Medical Association, 2016.

20. Poole JE, Gleva MJ, Mela T, et al. Complication rates associated with pacemaker or implantable cardioverter-defibrillator generator replacements and upgrade procedures. Circulation 2010;122:1553-61.

21. Daubert JP, Zareba W, Cannom DS, et al. Inappropriate implantable cardioverterdefibrillator shocks in MADIT II: frequency, mechanisms, predictors, and survival impact. J Am Coll Cardiol 2008:51:1357-65.

22. Takahashi T, Bhandari AK, Watanuki M, et al. High incidence of device-related and lead-related complications in the dual-chamber implantable cardioverter defibrillator compared with the single-chamber version. Circ J 2002;66:746-50.

23. Duray GZ, Schmitt J, Cicek-Hartvig S, et al. Complications leading to surgical revision in implantable cardioverter defibrillator patients: comparison of patients with single-chamber, dual-chamber, and biventricular devices. Europace 2009;11:297-302. 